\title{
Chlorogenic Acid Exerts Neuroprotective Effect Against Hypoxia-Ischemia Brain Injury in Neonatal Rats by activating Sirt1 to Regulate the Nrf2-NF-kB Signaling Pathway
}

\section{Yihui Zheng}

The Second Affiliated Hospital and Yuying Children's Hospital of Wenzhou Medical University https://orcid.org/0000-0003-0521-9389

Zhenlang Lin (D 13806689800@163.com )

The Second Affiliated Hospital and Yuying Children's Hospital of Wenzhou Medical University https://orcid.org/0000-0001-8068-5676

\section{Luyao Li}

The Second Affiliated Hospital and Yuying Children's Hospital of Wenzhou Medical University

\section{Binwen Chen}

The Second Affiliated Hospital and Yuying Children's Hospital of Wenzhou Medical University

\section{Yu Fang}

The Second Affiliated Hospital and Yuying Children's Hospital of Wenzhou Medical University

Wei Lin

The Second Affiliated Hospital and Yuying Children's Hospital of Wenzhou Medical University

\section{Tianlei Zhang}

The Second Affiliated Hospital and Yuying Children's Hospital of Wenzhou Medical University

\section{Xiaoli Feng}

The Second Affiliated Hospital and Yuying Children's Hospital of Wenzhou Medical University

\section{Xiaoyue Tao}

The Second Affiliated Hospital and Yuying Children's Hospital of Wenzhou Medical University

\section{Yiqing Wu}

The Second Affiliated Hospital and Yuying Children's Hospital of Wenzhou Medical University

\section{Xiaoqin Fu}

The Second Affiliated Hospital and Yuying Children's Hospital of Wenzhou Medical University

\section{Research}

Keywords: Chlorogenic acid, hie, Sirt1, Nrf2-NF-KB

Posted Date: October 5th, 2021 
DOl: https://doi.org/10.21203/rs.3.rs-882599/v1

License: (c) (1) This work is licensed under a Creative Commons Attribution 4.0 International License. Read Full License 


\section{Abstract}

Background: Neonatal hypoxic-ischemic brain injury (HIE) is caused by perinatal asphyxia, which is associated with various confounding factors. Although studies on the pathogenesis and treatment of HIE have matured, sub-hypothermia is the only clinical treatment available for HIE. Previous evidence indicates that chlorogenic acid (CGA) exerts a potential neuroprotective effect on brain injury. However, the role of CGA on neonatal $\mathrm{HI}$ brain damage and the exact mechanism remains elusive.

Here, we investigate the effects of CGA on $\mathrm{HI}$ models in vivo and in vitro and explore the underlying mechanism.

Methods: In the in vivo experiment, we ligated the left common carotid artery of 7-day-old rats and placed the rats in a hypoxic box for 2 hours. We did not ligate the common carotid artery of the pups in the sham group since they did not have hypoxia. Brain atrophy and infarct size were evaluated by Nissl staining, HE staining and 2,3,5-triphenyltetrazolium chloride monohydrate (TTC) staining. Morris Water Maze test (MWM) was used to evaluate neurobehavioral disorders. Western-blotting and immunofluorescence were used to detect the cell signaling pathway. Malondialdehyde (MDA) content test, catalase (CAT) activity detection and Elisa Assay was used to detect levels of inflammation and oxidative stress. In vitro experiments were performed on isolated primary neurons.

Result: In our study, pretreatment with CGA significantly decreased the infarct volume of neonatal rats after $\mathrm{HI}$, alleviated brain edema, and improved tissue structure in vivo. Moreover, we used the Morris water maze to verify CGA's effects on enhancing the learning and cognitive ability and helping to maintain the long-term spatial memory after HI injury. However, Sirt1 inhibitor EX-527 partially reversed these therapeutic effects. CGA pretreatment inhibited neuronal apoptosis induced by $\mathrm{HI}$ by reducing inflammation and oxidative stress. The findings suggest that CGA potentially activates Sirt1 to regulate the Nrf2-NF-KB signaling pathway by forming complexes thereby protecting primary neurons from oxygen-glucose deprivation (OGD) damage. Also, CGA treatment significantly suppresses HI-induced proliferation of glial.

Conclusion: Collectively, this study uncovered the underlying mechanism of CGA on neonatal HI brain damage. CGA holds promise as an effective neuroprotective agent to promote neonatal brain recovery from HI-induced injury.

\section{Background}

Neonatal hypoxic-ischemic encephalopathy is a brain injury that occurs in the perinatal period. Mounting evidence indicates that about 3 out of every 1,000 live births are likely to develop neonatal hypoxicischemic encephalopathy [1]. An ongoing concern is a high incidence and high mortality of HIE that needs to be solved urgently during the perinatal period [2]. Although scholars have deeply explored HIE, hypothermia treatment remains the only clinically recognized treatment method. Furthermore, the treatment window for mild hypothermia is short, and both the therapeutic effect and the nerve repair 
capacity for patients with severe neonatal ischemic hypoxic encephalopathy are limited [3]. Therefore, it would be imperative to uncover a safe and effective treatment method to assist in the diagnosis and treatment of brain injury with mild hypothermia therapy.

At low concentrations of antioxidants, an immature brain becomes highly susceptible to reactive oxygen and reactive nitrogen-mediated damage, and this is why the developing brain is extremely hypoxia sensitive. Studies have shown that neonatal hypoxic-ischemic encephalopathy involves different mechanisms, including oxidative stress [4], inflammation[5], apoptosis [6], autophagy[7], and so on. In particular, inflammation is implicated in the pathogenesis and pathophysiological changes of ischemic brain injury. Nuclear factor erythroid 2 related factor 2 (Nrf2), a key anti-inflammatory gene [8], directly regulates the expression of the HMOX1 gene encoded by the heme oxygenase HO-1 enzyme[9]. Evidence from in vitro and in vivo investigations has validated the role of the Nrf2-HO-1 signaling pathway in the inflammatory response. For instance, after Nrf2 knockdown, a study reported significantly increased expression of inflammation-related proteins COX-2, iNOS, IL-6, and TNF-a in the brains of Alzheimer's mice [10]. A similar investigation found elevated expression of HO-1 and NQO1 increased, providing evidence on the role of the Nrf2/HO-1 axis in inflammation [11]. Nuclear factor kappa-light-chainenhancer of activated B (NF-KB) interacts with Nrf2 during oxidative stress. As such, increased NF-KB nuclear displacement will promote the expression of pro-inflammatory factors IL-1, IL-6, and TNF-a. There are different mechanisms through which Nrf2 can negatively regulate the nuclear displacement of NFKB [12]. For instance, Nrf2 blocks the activation of NF-KB by reducing the level of intracellular ROS, and its up-regulation induces an increase in cellular $\mathrm{HO}-1$ level, decreasing the expression of related enzymes to prevent IKB-a proteasome degradation and inhibit the nuclear translocation of NF-KB [13-15]. Moreover, to prevent the binding of CBP to Nrf2, NF-KB reduce the amount of free state that is the active state of cAMP-response-element-binding protein-binding protein (CBP), the transcriptional coactivator of Nrf2 [16]. Sirtuins are a family of NAD-dependent protein deacetylases, that contribute to the regulation of various cellular mechanisms, including redox, inflammation, and autophagy. Recent experimental evidence indicate that SIRT1 play a part in the regulation of nuclear translocation of NF-kB, it activates the activation of Nrf2 and regulates the activity of NF-KB [17]. SIRT1 also regulates Nrf2 and inhibits the TLR4 pathway, thereby alleviating colitis-induced inflammation and pyrolysis [18].

Chlorogenic acid (CGA) is mainly extracted from coffee but also Chinese medicine honeysuckle, chrysanthemum, honeysuckle, and other plants [19]. It has anti-inflammatory and antioxidant effects, therefore, exert a protective effect in cardiovascular-related diseases [20]. CGA has also been shown to promote metabolism [21] and improve aging [22], which is why it is widely used in food and medicine. Evidence from studies indicates that CGA regulates the Nrf2 pathway related to oxidative stress in cerebral ischemia-reperfusion ( $\mathrm{Cl} / \mathrm{R})$ injury, exerting a neuroprotective effect [23]. In neonatal rats with alcoholic brain injury, CGA was revealed to exert an anti-apoptotic effect by regulating the enzyme activity of caspase-3 [24]. Elsewhere, CGA pretreatment increased copper and zinc superoxide dismutase (SOD1) in the hippocampus following transient forebrain ischemia and inhibited the expression of proinflammatory factors, interleukin-4 (IL-4) and interleukin-13 (IL-13) [25]. 
Although there is evidence that CGA plays a role in neuroprotection, studies on its effect in neonatal hypoxic-ischemic encephalopathy are immature, and the specific mechanism remains to be determined. Therefore, this study explored the role of CGA in HI-induced neonatal hypoxic-ischemic encephalopathy and elucidated whether the SIRT1/Nrf2/HO-1 pathway mediates this process.

\section{Materials And Methods}

\section{Reagents}

CGA (purity $\geq 98 \%$ ) was purchased from Solarbio (Wuhan, China). Primary antibodies were purchased as follows: TNF-a (ab66579; Abcam, Cambridge, United Kingdom), iNOS (ab178945; Abcam, Cambridge, United Kingdom), $\beta$-Actin (\#3700; Cell Signaling Technology, MA, United States), Histone H3 (\#4499; Cell Signaling Technology, MA, United States), IkBa (\#9242; Cell Signaling Technology, MA, United States), P65 (\#8242; Cell Signaling Technology, MA, United States), Nrf2 (\#12721; Cell Signaling Technology, MA, United States), HO-1 (\#43966; Cell Signaling Technology, MA, United States), IL-1ß (\#12703; Cell Signaling Technology, MA, United States), Sirt1 (DF6033, affinity, United States), SOD2 (AF5198, affinity, United States). The secondary antibodies of Goat Anti-Rabbit IgG and Alexa Fluor ${ }^{\circledR} 594$ labeled were purchased from Bioworld (OH, United States). Dimethylsulfoxide (DMSO) was purchased from Sigma Chemical Co. (St. Louis, MO, United States). Fetal bovine serum, B27, neurobasal medium, L-glutamine $(0.5 \mathrm{mM})$, and Dulbecco's modified Eagle medium were purchased from Gibco (Grand Island, NY, United States). Malondialdehyde (MDA) content test kit was purchased from Solarbio (Beijing, China). A catalase (CAT) activity detection kit was purchased from Solarbio (Beijing, China). Cell-Counting Kit-8 (CCK-8) was purchased from Dojindo (Kumano, Japan). The nuclear stain 4',6-diamidino-2- phenylindole (DAPI) was purchased from Beyotime (Shanghai, China). A nuclear and cytoplasmic protein extraction kit was purchased from Beyotime (Shanghai, China). Bovine serum albumin (BSA) was procured from Beyotime Biotechnology (Shanghai, China).

\section{Neonatal Hypoxic-Ischemic Brain Injury Model and Drug Administration}

Sprague Dawley (SD) rats (200-250g) were purchased from the Shanghai Zoological Center of the Chinese Academy of Sciences. Animal breeding and experiments were conducted as per the requirements of the Animal Breeding and Use Committee of Wenzhou Medical University. Adult SD rats mate freely, and male pups were used in the experiment on the $7^{\text {th }}$ day after birth (P 7). A modified Rice-Fannuzzi model was used as described previously [26], and isoflurane was applied to completely anesthetize and maintain $P 7$ pups. Subsequently, the left common carotid artery of $P 7$ young mice was separated within 5 min, ligated, and cut. The pups recovered with the mother 2 hours after the operation. The pups rested enough and were placed in a humidified mixed gas chamber composed of $92 \% \mathrm{~N}_{2}$ and $8 \% \mathrm{O}_{2}$ and ventilated at a flow rate of $3 \mathrm{~L} / \mathrm{min}$ for 2 hours. The above hypoxia equipment was placed in a constant temperature water bath at $37.5^{\circ} \mathrm{C}$. We did not ligate the common carotid artery of the pups in the sham operation group since they did not have hypoxia, but the common carotid artery of the pups of the other groups were ligated and the pups were treated separately. After the end of hypoxia, all the pups were 
caged to continue feeding, awaiting follow-up experiments. For the next few days, the rats were given drugs daily as follows: The CGA pretreatment group was intraperitoneally injected with different concentrations of CGA $(150,300$, or $600 \mathrm{mg} / \mathrm{kg})$ immediately after hypoxia, with an interval of 24 hours between each administration, and the young mice were euthanized to establish the most effective concentration. EX-527 $(0.5 \mathrm{mg} / \mathrm{kg}) 5 \mathrm{ul}$ each ( $2 \mathrm{~mm}$ rostral, $1.5 \mathrm{~mm}$ lateral to bregma, and $2.5 \mathrm{~mm}$ below the skull surface) was intracerebroventricularly injected, 30 minutes before hypoxia [27]. The needle was held for another 10 minutes after the injection is completed, and then withdrawn at a speed of 1 $\mathrm{mm} /$ minute.

\section{2,3,5-triphenyltetrazolium chloride (TTC) staining}

2,3,5-triphenyl tetrazolium chloride (TTC) staining method was employed to measure the volume of cerebral infarction based on the previous experimental procedure for assessing the area of cerebral infarction [28]. Brain tissues were collected from $\mathrm{P} 7$ young mice, 24 hours after $\mathrm{HI}$ injury, stored at $-20^{\circ} \mathrm{C}$ for 15 minutes, and cut into approximately $2 \mathrm{~mm}$ thick coronal sections. Then, the coronal brain slices were placed in $1 \% \operatorname{TTC}$ (Sigma, USA) solution and incubated in a $37^{\circ} \mathrm{C}$ oven in the dark for 30 minutes. Subsequently, the $11 \%$ TTC solution was discarded and the tissues were fixed overnight with $4 \%$ paraformaldehyde. Image-Pro Plus software was applied to measure the volume of cerebral infarction.

\section{Pathological staining}

Brain tissue specimens were collected 7 days after $\mathrm{HI}$ injury, whereby rats were anesthetized with isoflurane, and the heart was perfused with $25 \mathrm{ml}$ of sterile saline and further perfused with an equal volume of $4 \%$ paraformaldehyde. The brain specimen was immersed in $4 \%$ paraformaldehyde, stored at $4^{\circ} \mathrm{C}$ for 24 hours, and embedded in paraffin. Sections were cut at the thickness of $5 \mu \mathrm{m}$ from the paraffin block to visually show the hemispherical integrity of functional neurons between the cerebral cortex and the hippocampus coronal slices. The brain sections were then deparaffinized, hydrated, and stained with HE or Nissl solution (Sohrab, Beijing, China). Finally, an optical microscope was employed to assess and record the results of histological staining. ImageJ software was used to analyze the results.

\section{Nuclear protein and cytoplasmic protein extraction}

The nuclear protein was extracted for western blot detection of nuclear protein changes [29]. Appropriate amounts of $1 \mathrm{mM}$ PMSF cytoplasmic protein extraction reagents $A$ and $B$ were mixed at a 20:1 ratio to prepare a tissue homogenate, which was put in a homogenizer for tissue homogenization. Subsequently, the homogenized liquid was transferred to a $1.5 \mathrm{mlep}$ centrifuge tube, left to stand on ice for 15 minutes, and then centrifuged $\left(1,500 \mathrm{~g}\right.$ for 5 minutes at $\left.4^{\circ} \mathrm{C}\right)$. The supernatant (the cytoplasmic protein) was collected, and the pellet was stored at $-80^{\circ} \mathrm{C}$ waiting for further processing. The precipitate was added to the cytoplasmic protein extraction reagent A containing $1 \mathrm{mM}$ PMSF in a ratio of 1:10, shaken vigorously for 5 seconds, and left to stand on ice for 15 minutes. Following the addition of cytoplasmic protein extraction reagent $B$ at a ratio of 20:1, mixture was shaken vigorously for 5 seconds, left to stand on the ice for 1 minute, and centrifuged $\left(12,000 \mathrm{~g}\right.$ at $4^{\circ} \mathrm{C}$ for 5 minutes). The supernatant (cytoplasmic protein) 
was collected to save the pellet and merged into the previous cytoplasmic protein. After complete aspiration of the supernatant, $50 \mu \mathrm{l}$ of nuclear protein extraction reagent containing $1 \mathrm{mM}$ PMSF was added for precipitation. The mixture was vortexed for 30 seconds, then put in the ice bath, and vortexed again for $15-30$ seconds every 2 minutes for 30 minutes. The mixture was centrifuged at $12,000 \mathrm{~g}$ at $4^{\circ} \mathrm{C}$ for 10 minutes, and the supernatant was collected as the nuclear protein.

\section{Western Blot}

The extracted cerebral cortex tissue or primary neurons were lysed in RIPA lysis buffer containing $1 \mathrm{mM}$ PMSF. A tweezer was used to tear up the cerebral cortex tissue. The tissue was homogenized using a tissue homogenizer, fully sonicated on ice for 10 minutes, and then centrifuged $\left(4^{\circ} \mathrm{C}\right.$ at $12,000 \mathrm{rpm}$ at a speed of 20 minutes) to obtain the supernatant. Measurement of protein concentration was taken using the BCA kit (Beyotime) and the protein was prepared. Sodium dodecyl sulfate (SDS)-polyacrylamide separation gel electrophoresis was used to separate the protein, and then the bands were transferred to PVDF membrane (Millipore). After blocking with a $5 \%$ skimmed milk solution or a $5 \%$ BSA solution diluted with TBST for 4 hours, the membrane was incubated with primary antibodies overnight in a refrigerator at

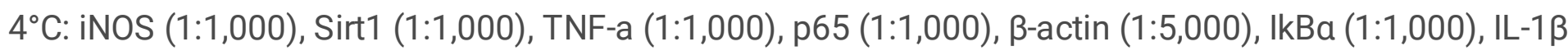
$(1: 1,000)$, Histone H3 (1:1,000), Nrf2 (1:1,000), and HO-1 (1:1,000). Subsequently, TBST was washed thrice for 5 minutes each time and incubated with the corresponding secondary antibody $(1: 10,000)$ for 90 minutes. Afte washing with TBST for 3 times, the blots were visualized using an ECL Plus chemiluminescence reagent kit (RPN3243; Amersham Bioscience, Bensenville, IL, USA) and quantified by the Imaging System (Bio-Rad).

\section{Elisa Assay}

We washed rat brain tissue with PBS, added PBS at a ratio of 1:9 and homogenized the samples on ice. We centrifuged the homogenate obtained above at $12,000 \mathrm{rpm}$ for 10 minutes at $4^{\circ} \mathrm{C}$, and took the supernatant $(100 \mu \mathrm{l})$ for analysis. The enzyme-linked immunosorbent assay (ELISA) (E-EL-R0012c, Elabscience) was used to detect the content of IL-1 $\beta$ in the brain tissue of rats in each group. We set up blank wells, standard wells and sample wells on the ELISA plate. We filled the blank well with $100 \mu \mathrm{l}$ of standard/sample diluent. The standard wells contained $100 \mu \mathrm{l}$ of standard samples diluted in multiples, and the sample wells contained $100 \mu$ of experimental samples. Then the plate was incubated at $37^{\circ} \mathrm{C}$ for 90 minutes. After being added the biotinylated antibody working solution $(100 \mu \mathrm{l} /$ well), the plate was incubated at $37^{\circ} \mathrm{C}$ for 60 minutes, followed by being washed with washing solution 3 times. Then the plate was incubated at for 30 min with $100 \mu$ l horseradish peroxidase conjugate solution. Next we washed it with washing solution for 5 times, then added $90 \mu$ of substrate solution to each well, and incubated it for 15 minutes at $37^{\circ} \mathrm{C}$. Finally, we added $50 \mu$ of stop solution, and immediately measured the optical density of IL-1 $\beta$ at $450 \mathrm{~nm}$ wavelength.

\section{Malondialdehyde (MDA) content test}


Lipid peroxidation level was detected based on the level of MDA [30]. Rats were euthanized after deep anesthesia with isoflurane three days after the $\mathrm{HI}$ injury, and the cerebral cortex tissues of the young rats were isolated and stored at $-80^{\circ} \mathrm{C}$ for subsequent analysis. We weighed about $0.1 \mathrm{~g}$ of tissue of each group, added $1 \mathrm{~mL}$ of extracting solution to homogenize in ice bath, centrifuged it at $8000 \mathrm{~g}$ at $4{ }^{\circ} \mathrm{C}$ for $10 \mathrm{~min}$, and took the supernatant for next testing. Subsequently, the MDA was extracted and placed in a microplate reader to detect the absorbance at wavelengths of $450 \mathrm{~nm}, 532 \mathrm{~nm}$, and $600 \mathrm{~nm}$. Finally, according to the MDA content (nmol/g mass) $=5 \times(12.9 \times(\triangle \mathrm{A} 532-\triangle \mathrm{A} 600)-2.58 \times \triangle \mathrm{A} 450) \div \mathrm{W}$. W: sample mass, g; $\Delta \mathrm{A} 450=\Delta 450$ measurement $-\Delta 450$ blank; $\Delta \mathrm{A} 532=\Delta 532$ measurement $-\Delta 532$ blank; $\Delta \mathrm{A} 600=\Delta 600$ measurement- $\Delta 600$ blank, we measured the concentration of MDA.

\section{Catalase (CAT) activity detection}

Catalase (CAT) is the most important hydrogen peroxide scavenging enzyme in the body [31, 32]. Using the catalase (CAT) activity detection kit as per the manufacturer's instructions and a microplate reader, we detected the CAT content according to the change in absorbance over time at a wavelength of $240 \mathrm{~nm}$.

\section{Immunofluorescence staining}

Coronal brain tissue slices were stained with GFAP. Briefly, the slices were dried in an oven at $65^{\circ} \mathrm{C}$ for 3 hours and immersed in xylene to deparaffinize for 20 minutes. The slices were hydrated in gradient alcohol and washed thrice with PBS (5 minutes each time). Following antigen retrieval with sodium citrate, slices were washed thrice with PBS (5 minutes each time), diluted with $0.3 \%$ Triton X-100 (PBS), and reacted for 15 minutes at room temperature. We washed slices 3 times with PBS for 5 minutes each time, blocked them with $10 \%$ goat serum (PBS), and then incubated slices overnight at $4^{\circ} \mathrm{C}$ with GFAP (1:200) primary antibody. On the second day, after 3 times wash with PBS, slices were exposed to a secondary antibody (1:200) labeled with Alexa Fluor ${ }^{\circledR} 594$ for 1.5 hours. After washing thrice with PBS (5 minutes each time), they were incubated with DAPI for 10 minutes, washed with PBS, and mounted with an anti-quenching agent. The samples were taken and preserved in an Olympus fluorescence microscope (Tokyo, Japan). ImageJ software was employed to determine the fluorescence intensity.

\section{Morris Water Maze test (MWM)}

The Morris Water Maze Test (MWM) is a behavioral experiment for determining the cognitive function of animals [33]. We applied the MWM test, 21 days after the $\mathrm{HI}$ injury, to evaluate the learning and memory abilities of experimental animals, whereby they were put to swim to find a platform hidden underwater. A black circular pool with a diameter of $140 \mathrm{~cm}$ and a height of $50 \mathrm{~cm}$ was prepared in a room protected from noise and light. The water depth of the pool was $1 \mathrm{~cm}$ higher than the movable platform. Water was dyed black with non-toxic black ink and the pool was divided into four equal quadrants. The rats were trained for 6 days, after which the platform was removed. The swimming route, incubation time, and the number of times on stage were recorded. The experiment was performed by the SLY-WMS Morris water maze experiment system. 


\section{Primary cortical neuron extraction and culture}

Primary cortical neurons were extracted from the cerebral cortex of SD neonatal rats (P 0). Briefly, the newborn rat was immersed in $75 \%$ alcohol for 20 minutes, the brain was isolated to separate the cerebral cortex and washed thrice with PBS. Then we used $0.25 \%$ papain-EDTA solution to digest cerebral cortex tissue at $37^{\circ} \mathrm{C}$ for 15 minutes. After centrifugation of the final product above, the pellet was introduced to a 6-well cell culture plate coated with poly-D-lysine according to $1 \times 105$, incubated $\left(5 \% \mathrm{CO}_{2}\right.$ at $37^{\circ} \mathrm{C}$ for 6 hours). The culture medium was replaced with $2 \%$ B27, 0.5 Neural basal medium of mM L-glutamine, penicillin/streptomycin antibiotics.

\section{Cell viability}

The cytotoxicity of CGA to primary cortical neurons was detected by the CCK-8 kit. Briefly, primary cortical neurons were extracted and inoculated in 96 -well plates $(8,000$ cells/well). Then, the cells were treated with CGA in a concentration gradient of $0,100,200,300$, and 400nM for 24 hours. Finally, $10 \mu$ l of CCK-8 solution was added to each well of the 96 -well plate and incubated at $37^{\circ} \mathrm{C}$ for 1.5 hours. A microplate reader (Leica Microsystem, Germany) was used to detect the absorbance of each well at a wavelength of $450 \mathrm{~nm}$.

\section{Results}

\section{Chlorogenic acid attenuates hypoxic-ischemic brain injury in neonatal rats}

Rats were intraperitoneally injected with CGA at the range of concentrations $(150,300,600 \mathrm{mg} / \mathrm{kg})$, every 24 hours for 3 consecutive days to investigate the role of CGA in the HI brain injury process and to establish the most effective animal administration dose. According to TTC staining results and the quantitative analysis of cerebral infarction volume, the $150,300,600 \mathrm{mg} / \mathrm{kg}$ doses of CGA effectively decreased the volume of cerebral infarction (Figures 1a-b); notably, the therapeutic effect increased with an increase in the concentration. Therefore, we selected a concentration of $600 \mathrm{mg} / \mathrm{kg}$ for subsequent experiments. At the same time, the general brain changes 7 days after the $\mathrm{HI}$ injury, were assessed and compared among groups. The HI group was characterized by more edema and liquefaction as compared to the sham operation group (Figure 1c-d). Furthermore, brain damage in the CGA $(600 \mathrm{mg} / \mathrm{kg}$ ) pretreatment group was significantly less compared to the $\mathrm{HI}$ injury group (Figure $1 \mathrm{~d}$ ). These data strongly demonstrate that CGA pretreatment can alleviate the brain damage of newborn rats after $\mathrm{HI}$ injury.

\section{Chlorogenic acid-induced protection of brain post-hypoxic-ischemic brain injury by decreasing inflammation and oxidative stress}

Previous evidence indicates that CGA can promote the body's anti-inflammatory and anti-oxidative stress effects. Here, using ELISA, we detected pro-inflammatory factor IL-1 $\beta$ secreted in brain tissue. Results demonstrated significantly increased IL-1 $\beta$ levels after HI injury. Notably, after CGA injection, we observed 
significantly reduced IL-1 $\beta$ secretion in brain tissue (Figure 2a). Moreover, the level of lipid oxidation in the brain tissue was detected using the MDA kit. It was revealed that the level of MDA in the brain tissue of the sham operation group was $63.92 \pm 10.37 \mathrm{nmol} / \mathrm{g}$, while the average level of MDA in the brain tissue of the $\mathrm{HI}$ group was $98.48 \pm 10.01 \mathrm{nmol} / \mathrm{g}$. Moreover, the average level of MDA in the brain tissue of the CGA pretreatment group was $83.44 \pm 10.00 \mathrm{nmol} / \mathrm{g}$ (Figure $2 \mathrm{~b}$ ). CAT detection of the level of $\mathrm{H}_{2} \mathrm{O}_{2}$ elimination enzymes showed that the level of CAT (catalase) in the brain tissue of the sham operation group was $387.25 \pm 59.61 \mathrm{U} / \mathrm{g}$, the level of CAT in the brain tissue of the HI group was $168.58 \pm 50.29 \mathrm{U} / \mathrm{g}$, and the level of CAT in the brain tissue of the CGA pretreatment group was $278.16 \pm 67.07 \mathrm{U} / \mathrm{g}$ (Figure 2C). The tissue proteins of the cerebral cortex of young rats were extracted 3 days after injury, followed by western blot detection of the expression levels of pro-inflammatory factors IL-1 $\beta$, iNOS, TNF-a, and SOD2/MnSOD. Results demonstrated that, after $\mathrm{HI}$ injury, the levels of pro-inflammatory factors IL-1 $\beta$, iNOS, and TNF- $a$ increased significantly, while the levels of SOD2/MnSOD decreased significantly. Compared with the HI injury group, the CGA group exhibited significantly lower levels of pro-inflammatory factors IL-1 $\beta$, iNOS, and TNF- $a$ and significantly higher levels of SOD2/MnSOD. These data demonstrate the potential neuroprotective effects of CGA by reducing inflammation and oxidative stress (Figures $2 \mathrm{~d}-\mathrm{h}$ ).

\section{Chlorogenic acid exerts a neuroprotective effect in hypoxic-ischemic brain injury by activating Sirt1 to regulate the Nrf2-NF-KB signaling pathway}

Studies have revealed that CGA exerts anti-inflammatory effects by activating Nrf2-NF-KB during cerebral ischemia-reperfusion injury[34]. Sirt1 is a key regulator of the Nrf2-NF-KB signaling pathway. We explored the effect of CGA on Nrf2-NF-KB via Western blot analysis. Nuclear protein detection results indicated that $\mathrm{HI}$ damage could promote the nuclear transfer of Nrf2, and increase the nuclear levels level of NF-KB (Figures 3a, e-f). In the CGA pretreatment group, the nuclear levels level of Nrf2 was higher than that of the HI group, while the levels level of NF-KB in the nucleus was significantly lower than the HI group. Cytoplasmic protein analysis demonstrated that, in the $\mathrm{HI}$ group, the levels of $\mathrm{HO}-1$ increased while the levels level of Sirt1 and ІкB decreased significantly. The levels of HO-1, Sirt1, and IKB were all higher in the CGA pretreatment group as compared to those of the $\mathrm{HI}$ injury group (Figures 3a-d). After using the selective inhibitor of Sirt1, EX-527 in the pretreatment group, the level of nuclear internalization of Nrf2 was significantly lower than that of the CGA pretreatment group. And the level of nuclear internalization of NF-KB was higer than that of the CGA pretreatment group. The selective inhibitor of Sirt1, EX-527, counteracted the neuroprotective effect of CGA through Nrf2-NF-KB (Figures 3g-i).

\section{Chlorogenic acid attenuates apoptosis induced by $\mathrm{HI}$ injury}

To detect the changes in the levels of apoptosis in each group, total protein was extracted from each group $24 \mathrm{~h}$ after $\mathrm{HI}$, followed by western blot detection of the ratio of $\mathrm{Bcl} / 2 / \mathrm{Bax}$ in each group. Results showed significantly reduced Bcl2/Bax in the HI group. Significantly, the Bcl2/Bax ratio of the CGA treatment group was higher as compared to that of the HI group. The selective inhibitor of Sirt1, EX-527, reversed the anti-apoptotic effect of CGA treatment (Figures 4a-b). 


\section{Chlorogenic acid improves hypoxic-ischemic brain injury-induced brain tissue structural damage}

The protective effect of CGA on neurons was assessed via HE staining and Nissl staining. By comparing the Nissl bodies of the $\mathrm{HI}$ injury group and the sham operation group, the cerebral cortex and hippocampus (DG area, CA1 area, CA3 area) of the sham operation group had large Nissl bodies mostly arranged close to the nucleus, while the HI group was almost invisible to Nissl body (Figure 5a). Assessment of the stained sections of pathological tissues revealed an apparent left hemibrain injury in the $\mathrm{HI}$ injury group, especially in the cerebral cortex and hippocampus (DG area, CA1 area, CA3 area), the cell arrangement was disordered, and most neurons died. Compared the number of normal neurons in the selected area of the cerebral cortex in each group, we observed there were almost no normal neurons in the $\mathrm{HI}$ injury group. Moreover, the number in the images of hippocampal DG region, CA1 region, and CA3 region were counted and the number of cells in the DG, CA1 and CA3 areas decreased significantly after $\mathrm{HI}$ injury. CGA treatment significantly increased the number of normal neurons and Nissl bodies with the increase in concentration, and a gradual recovery of the changes in cell structure was reported with the increase in dose. Of note, the selective inhibitor of Sirt1, EX-527, reversed the therapeutic effect of CGA (Figure 5b-f).

\section{Chlorogenic acid treatment suppresses HI-induced activation of astrocyte in the neonate brain}

GFAP is a crucial indicator of astrocyte activation. Here, GFAP expression in each group was assessed by tissue immunofluorescence staining. Experimental results demonstrated significantly high expression of GFAP in the cerebral cortex and hippocampal CA3 area after $\mathrm{HI}$ injury, and the astrocytes in these two areas were significantly enlarged and densely distributed, and the cell branches increased. In the HI+CGA group, GFAP expression was significantly reduced, the activation of astrocytes was blocked, and the branches of astrocytes decreased (Figure 6a-b). At the same time, western blot detection of cerebral cortex tissue proteins, 24 hours after $\mathrm{HI}$ injury, revealed that GFAP levels after HI+CGA treatment was consistent with the results in immune tissue fluorescence (Figure 6c-d).

\section{Chlorogenic acid ameliorates the long-term learning and cognitive function of rats with hypoxic-ischemic brain injury}

We evaluated the effect of CGA pretreatment on learning and cognitive function after $\mathrm{HI}$ injury by conducting the Morris water maze experiment. Data acquired in the spatial acquisition test revealed that after $\mathrm{HI}$ injury, the learning ability of rats decreased and the average escape latency of $\mathrm{HI}$ injury was longer as compared to that of the sham group. However, the average escape latency of rats in the $\mathrm{HI}+$ CGA group was significantly shortened, a trend that could be reversed by EX-527, the selective inhibitor of Sirt1 (Figure 7a-b). In the reference memory test, the platform was removed after the last training on the $6^{\text {th }}$ day. After 24 hours, to investigate the spatial memory ability of the rats, we counted the number of times the rats crossed the platform after the platform was removed. In the CGA pretreatment group, we reported a significant increase in the number of bench crossings; however, EX-527 could reduce this effect (Figure 7c-d). 


\section{Chlorogenic acid decreases inflammation and oxidative stress in primary cortical neurons, induced by oxygen and glucose deprivation}

To ascertain the in vivo mechanism underlying the above-mentioned phenomenon, we constructed an in vitro primary neuron OGD model. Oxygen-sugar deprivation of primary neurons was achieved on the $7^{\text {th }}$ day of culture in vitro for 2 hours, followed by administration of different doses of CGA $(0,100,200,300$ and $400 \mu \mathrm{M}$ ) after OGD. After 24 hours of reoxygenation, neuronal proliferation activity was detected by the CCK8 assay. Results revealed that different drug concentrations did not affect neuronal cells under normal circumstances. Notably, after OGD, the proliferative activity of the cells was reduced to approximately $47.5 \%$. By increasing the CGA concentration to $200,300 \mu \mathrm{M}$, the cell proliferation activity was improved significantly. No significant difference in proliferative activity was reported at concentrations of $200 \mu \mathrm{M}$ and $300 \mu \mathrm{M}$; as such, we selected $200 \mu \mathrm{M}$ as the therapeutic concentration for in vitro experiments (Figure 8a-b).

Through western blot, we detected the levels levels of pro-inflammatory factors IL-1 $\beta$, iNOS, TNF-a, and antioxidant-related factors SOD2/MnSOD in the total protein of primary neurons. The experimental results revealed that, after oxygen-sugar deprivation, the levels of pro-inflammatory factors IL-1 $\beta$, iNOS, and TNF-a increased significantly, while the levels of SOD2/MnSOD decreased significantly. Compared to the OGD group, the levels of pro-inflammatory factors IL-1 $\beta$, iNOS, and TNF-a reduced significantly in the CGA $(200 \mu \mathrm{M})$ pretreatment group, while the levels of SOD2/MnSOD increased significantly. These data provide evidence on the neuroprotective effect of CGA by reducing inflammation and oxidative stress (Figure 8d-h).

Moreover, after CGA pretreatment, the levels level of Nrf2 in the nucleus was higher than that of the control group. And the levels level of NF-KB in the nucleus was significantly reduced in the CGA pretreatment group. The cytoplasmic protein test results showed that, after OGD, HO-1 was up-regulated, while the levels levels of Sirt1 and ІкB were significantly reduced. However, CGA pretreatment significantly increased the levels level of H01, Sirt1 and IKB (Figure 9a-d). By introducing EX-527, a selective inhibitor of Sirt1, western blot detection of the levels of neuronal apoptosis-related proteins demonstrated a reversed anti-apoptotic effect of CGA through Nrf2-NF-KB (Figure 9a, e-f). The cell viability analysis after pretreatment of primary neurons with EX-527verified that EX-527 reversed the protective effect of CGA (Figure 8c).

\section{Discussion}

Neonatal hypoxic-ischemic encephalopathy (HIE) is a type of perinatal asphyxia-induced brain damage. Although research on the pathogenesis of HIE has matured, mild hypothermia remains the sole clinical treatment for HIE. The short time window of mild hypothermia treatment and the limited nerve repair effect of severe HIE warrants an urgent exploration of an auxiliary treatment method for mild hypothermia to reduce HIE-induced nerve damage. 
The research on the pathological damage mechanism of HIE covers cell inflammation, oxidative stress, apoptosis, autophagy, among others. It is of particular note that inflammation and oxidative stress are key players in HIE treatment. Studies by Serafina Perrone et al. demonstrated that hypoxic-ischemic injury activates various inflammation-related pathways, and a variety of inflammation-related cellular molecules, including cytokines (IL-1 $\beta$, IL-6, IL-8, IL-10, TNF - $a$, etc) are activated after HI injury [35, 36]. Stress damage such as hypoxia and ischemia have been revealed to potentially cause significant changes in the levels of HO-1, HIF-1 a, and other hypoxia-related proteins in brain tissue which were consistent with the results of the pro-inflammatory factors TNF $-a$ and IL-1 $\beta$ in this experiment in the $\mathrm{HI}$ group. [35]. In vitro studies have revealed that the addition of the pro-inflammatory factor IL-1 $\beta$ inhibits the levels of HO-1 and can promote the occurrence of inflammation. Schipper et al. showed that upregulation of HO-1 levels in the pathogenesis of multiple sclerosis (MS) was beneficial in maintaining the stability of the microenvironment in the cells and play a protective role after autoimmune neuroinflammation[37]. Nuclear factor erythroid 2 related factor 2 (Nrf2) is the upstream molecule of HO1 and is activated by nuclear transfer [38]. Nerve injury causes up-regulation of Nrf2. Therefore, by regulating Nrf2 levels, the downstream cascade is activated to relieve the body's oxidative stress and inflammation [39]. In recent years, some anti-oxidative stress and anti-inflammatory drugs such as licochalcone A, Lycium ruthenicum polysaccharide 3 (LRP3) have been revealed to up-regulate Nrf2 intranuclear metastasis to exert neuroprotective effects, which is consistent with our present findings[40, 41]. NF-kB is an important nuclear transcription factor in cells. Kauppinen et al. found that inhibition of silent information regulator 1 (Sirt1) altered energy metabolism in cells, further stimulating the NF-KBinduced inflammatory response [42]. Our research results and the above research results can be mutually verified.

Chlorogenic acid (CGA) is a phenolic antioxidant mainly extracted from honeysuckle. Previous evidence indicates that CGA exerts a neuroprotective effect in neurological diseases, including cerebral ischemia/reperfusion injury. For instance, Oboh et al. demonstrated that CGA exerted a neuroprotective effect in Alzheimer's disease by inhibiting the activities of acetylcholinesterase (AChE) and butyrylcholinesterase (BChE) and reducing the decomposition of acetylcholine and butyrylcholine [43]. Elsewhere, Liu et al. demonstrated that CGA regulated the Nrf2 pathway and further up-regulated Nrf2, NQO-1, and HO-1 to reverse cerebral ischemia/reperfusion-induced brain damage [23]. Shah et al. also found that CGA inhibited oxidative stress by reducing the level of reactive oxygen species (ROS), and further reduced focal cerebral ischemia-induced neuronal cell apoptosis [44]. Here, we, for the first time, proved that CGA exerts a neuroprotective effect on neonatal hypoxic-ischemic brain damage. Through in vivo and in vitro experiments, we have confirmed that CGA exerts anti-inflammatory effects by regulating Sirt1 to activate Nrf2-NF-kB, and further decreased the apoptosis of brain neurons. At the same time, we investigated the role of Sirt1 in the Nrf2-NF-KB pathway through intracerebroventricular injection in vivo and the addition of Sirt1 selective inhibitor EX-527 in vitro. Our analysis revealed that EX-527 reversed the aforementioned protective effects. Through investigation of learning and cognitive functions, the results strongly demonstrated that CGA improves the spatial memory and learning and cognitive abilities of the $\mathrm{HI}$-injured brain. The activation of astrocytes represents the proliferation of brain glial, which is a key 
contributor to brain tissue damage. Mounting evidence has also demonstrated the potential role of GFAP in the activation process of astrocytes in hypoxia, ischemia, and lipid peroxidation damage $[45,46]$. Collectively, CGA plays a neuroprotective function in neonatal hypoxic-ischemic brain injury.

\section{Declarations}

\section{Acknowledgments}

Not applicable.

\section{Authors' contributions}

$Y Z, X F$ and $Z L$ designed the research study. $Y Z, B C, W L$, and $T Z$ found and read relevant literatures. $Y Z$, $X F$ and $Z L$ designed the experiments. $Y Z, B C$, $L L$ and $Y F$ performed experiments. $Y Z, X F, X T$ and $Y W$ analyzed data to form graphs. $Y Z$ wrote the manuscript. XF and $Z L$ helped to modified the manuscript. All authors have read and agreed to the published version of the manuscript.

\section{Funding}

The study was funded by Basic Medical and Health Technology Project of Wenzhou Science and Technology Bureau (No. Y20190001) and the National Natural Science Fund (No. 81771624).

\section{Availability of data and materials}

Not applicable.

\section{Ethics approval and consent to participate}

This study was reviewed and approved by the Animal Care and Use Committee of Wenzhou Medical University.

\section{Consent for publication}

Not applicable.

\section{Competing interests}

None of the authors of this manuscript has any conflict of interest to declare.

\section{References}

1. Mietzsch $\mathrm{U}$, et al. Active cooling temperature required to achieve therapeutic hypothermia correlates with short-term outcome in neonatal hypoxic-ischaemic encephalopathy. J Physiol. 2020;598(2):415-24. 
2. Rocha-Ferreira E, et al. Curcumin: Novel Treatment in Neonatal Hypoxic-Ischemic Brain Injury. Frontiers in physiology. 2019;10:1351.

3. Oorschot D, Sizemore R, Amer A. Treatment of Neonatal Hypoxic-Ischemic Encephalopathy with Erythropoietin Alone, and Erythropoietin Combined with Hypothermia: History, Current Status, and Future Research. International journal of molecular sciences, 2020. 21(4).

4. Hu X, et al., Rh-CSF1 Attenuates Oxidative Stress and Neuronal Apoptosis via the CSF1R/PLCG2/PKA/UCP2 Signaling Pathway in a Rat Model of Neonatal HIE. Oxidative medicine and cellular longevity, 2020. 2020: p. 6801587.

5. Chen Z, et al. MicroRNA-374a-5p inhibits neuroinflammation in neonatal hypoxic-ischemic encephalopathy via regulating NLRP3 inflammasome targeted Smad6. Life sciences. 2020;252:117664.

6. Si W, et al., $\beta A T 1 R / G S K-3 / m$ TOR Signaling Pathway Involved in Angiotensin II-Induced Neuronal Apoptosis after HIE Both In Vitro and In Vivo. Oxidative medicine and cellular longevity, 2020. 2020: p. 8864323.

7. Ye L, et al. CpG-ODN exerts a neuroprotective effect via the TLR9/pAMPK signaling pathway by activation of autophagy in a neonatal HIE rat model. Exp Neurol. 2018;301:70-80.

8. Luo X, et al. N-acetylserotonin Derivative Exerts a Neuroprotective Effect by Inhibiting the NLRP3 Inflammasome and Activating the PI3K/Akt/Nrf2 Pathway in the Model of Hypoxic-Ischemic Brain Damage. Neurochem Res. 2021;46(2):337-48.

9. Loboda A, et al. Role of Nrf2/HO-1 system in development, oxidative stress response and diseases: an evolutionarily conserved mechanism. Cell Mol Life Sci. 2016;73(17):3221-47.

10. Ren P, et al., Nrf2 Ablation Promotes Alzheimer's Disease-Like Pathology in APP/PS1 Transgenic Mice: The Role of Neuroinflammation and Oxidative Stress. Oxidative medicine and cellular longevity, 2020. 2020: p. 3050971.

11. Liu Q, et al. Antioxidant effects of ginkgolides and bilobalide against cerebral ischemia injury by activating the Akt/Nrf2 pathway in vitro and in vivo. Cell stress chaperones. 2019;24(2):441-52.

12. Wardyn J, Ponsford A, Sanderson C. Dissecting molecular cross-talk between Nrf2 and NF-KB response pathways. Biochemical Society transactions. 2015;43(4):621-6.

13. Martorana F, et al., KDifferential Modulation of NF-B in Neurons and Astrocytes Underlies Neuroprotection and Antigliosis Activity of Natural Antioxidant Molecules. Oxidative medicine and cellular longevity, 2019. 2019: p. 8056904.

14. Subedi L, et al., Anti-Inflammatory Effect of Sulforaphane on LPS-Activated Microglia Potentially through JNK/AP-1/NF-KB Inhibition and Nrf2/HO-1 Activation. Cells, 2019. 8(2).

15. Kim K, et al. Terrein suppressed lipopolysaccharide-induced neuroinflammation through inhibition of NF-KB pathway by activating Nrf2/HO-1 signaling in BV2 and primary microglial cells. J Pharmacol Sci. 2020;143(3):209-18.

16. Katoh Y, et al., Two domains of Nrf2 cooperatively bind CBP, a CREB binding protein, and synergistically activate transcription. Genes to cells: devoted to molecular \& cellular mechanisms, 
2001. 6(10): p. 857-68.

17. Arioz B, et al., Melatonin Attenuates LPS-Induced Acute Depressive-Like Behaviors and Microglial NLRP3 Inflammasome Activation Through the SIRT1/Nrf2 Pathway. Frontiers in immunology, 2019. 10: p. 1511.

18. Yin Y, et al. Curcumin improves necrotising microscopic colitis and cell pyroptosis by activating SIRT1/NRF2 and inhibiting the TLR4 signalling pathway in newborn rats. Innate immunity. 2020;26(7):609-17.

19. Santana-Gálvez J, Cisneros-Zevallos L, Jacobo-Velázquez D, Chlorogenic Acid: Recent Advances on Its Dual Role as a Food Additive and a Nutraceutical against Metabolic Syndrome. Molecules (Basel, Switzerland), 2017. 22(3).

20. Agunloye 0 , et al. Cardio-protective and antioxidant properties of caffeic acid and chlorogenic acid: Mechanistic role of angiotensin converting enzyme, cholinesterase and arginase activities in cyclosporine induced hypertensive rats. 109: Biomedicine \& pharmacotherapy = Biomedecine \& pharmacotherapie; 2019. pp. 450-8.

21. Bhandarkar N, Brown L, Panchal S. Chlorogenic acid attenuates high-carbohydrate, high-fat dietinduced cardiovascular, liver, and metabolic changes in rats. 62. New York: Nutrition research; 2019. pp. 78-88.

22. Gao L, et al., Chlorogenic Acid Alleviates A mTOR/TFEB Signaling Pathway. Drug design, development and therapy, 2020. 14: p. 1705-1716.

23. Liu D, et al., Protective Effects of Chlorogenic Acid on Cerebral Ischemia/Reperfusion Injury Rats by Regulating Oxidative Stress-Related Nrf2 Pathway. Drug design, development and therapy, 2020. 14: p. 51-60.

24. Fang S, et al. Beneficial effects of chlorogenic acid on alcohol-induced damage in PC12 cells. 79: Biomedicine $\&$ pharmacotherapy $=$ Biomedecine $\&$ pharmacotherapie; 2016. pp. 254-62.

25. Lee T, et al., Experimental Pretreatment with Chlorogenic Acid Prevents Transient Ischemia-Induced Cognitive Decline and Neuronal Damage in the Hippocampus through Anti-Oxidative and AntiInflammatory Effects. Molecules (Basel, Switzerland), 2020. 25(16).

26. Vannucci R, Vannucci S. Perinatal hypoxic-ischemic brain damage: evolution of an animal model. Developmental neuroscience. 2005;27:81-6.

27. Ye L, et al. FGF21 promotes functional recovery after hypoxic-ischemic brain injury in neonatal rats by activating the PI3K/Akt signaling pathway via FGFR1/ $\beta$-klotho. Exp Neurol. 2019;317:34-50.

28. Rong Z, et al. Hesperidin pretreatment protects hypoxia-ischemic brain injury in neonatal rat. Neuroscience. 2013;255:292-9.

29. Sheng $\mathrm{F}$, et al. Increased expression and activity of MMP-9 in C-reactive protein- induced human THP1 mononuclear cells is related to activation of nuclear factor kappa-B. Journal of Huazhong University of Science and Technology. Medical sciences = Hua zhong ke ji da xue xue bao. Yi xue Ying De wen ban = Huazhong keji daxue xuebao. Yixue Yingdewen ban. 2009;29(4):399-403. 
30. Peng Q, et al. Inhibiting the CD38/CADPR pathway protected rats against sepsis associated brain injury. Brain research. 2018;1678:56-63.

31. Yang $Y$, et al. Amelioration of nonalcoholic fatty liver disease by swertiamarin in fructose-fed mice. Phytomedicine: international journal of phytotherapy phytopharmacology. 2019;59:152782.

32. Yu F, Tong L, Cai D. Sevoflurane inhibits neuronal apoptosis and expressions of HIF-1 and HSP70 in brain tissues of rats with cerebral ischemia/reperfusion injury. Eur Rev Med Pharmacol Sci. 2020;24(9):5082-90.

33. Xiao Q, et al. MiR-410-3p overexpression ameliorates neurological deficits in rats with hypoxicischemic brain damage. Brain research bulletin. 2020;162:218-30.

34. Liu D, et al. Protective Effects of Chlorogenic Acid on Cerebral Ischemia/Reperfusion Injury Rats by Regulating Oxidative Stress-Related Nrf2 Pathway. 14: Drug Design, Development and Therapy; 2020.

35. Schipper HM, et al. The Sinister Face of Heme Oxygenase-1 in Brain Aging and Disease. 172: Progress in Neurobiology; 2018.

36. Massaro AN, et al. Plasma Biomarkers of Brain Injury in Neonatal Hypoxic-Ischemic Encephalopathy. J Pediatr. 2018;194:67-75.

37. Hauptmann J, et al., Interleukin-1 promotes autoimmune neuroinflammation by suppressing endothelial heme oxygenase-1 at the blood-brain barrier. Acta Neuropathologica, 2020(Pt A).

38. Bian $\mathrm{H}$, et al., Dihydrolipoic acid protects against lipopolysaccharide-induced behavioral deficits and neuroinflammation via regulation of Nrf2/HO-1/NLRP3 signaling in rat. Journal of Neuroinflammation, 2020. 17(1).

39. Gao Y, et al. Resveratrol mitigates the oxidative stress mediated by hypoxic-ischemic brain injury in neonatal rats via Nrf2/HO-1 pathway. Pharm Biol. 2018;56(1):440-9.

40. Liu $X$, et al. Neuroprotective effect of licochalcone A against oxygen-glucose deprivation/reperfusion in rat primary cortical neurons by attenuating oxidative stress injury and inflammatory response via the SIRT1/Nrf2 pathway. Journal of cellular biochemistry. 2018;119(4):3210-9.

41. Deng K, et al. Lycium ruthenicum Murr polysaccharide protects cortical neurons against oxygenglucose deprivation/reperfusion in neonatal hypoxic-ischemic encephalopathy. Int J Biol Macromol. 2020;158:562-8.

42. Kauppinen A, et al. Antagonistic crosstalk between NF-KB and SIRT1 in the regulation of inflammation and metabolic disorders. Cellular signalling. 2013;25(10):1939-48.

43. Oboh G, et al. Comparative study on the inhibitory effect of caffeic and chlorogenic acids on key enzymes linked to Alzheimer's disease and some pro-oxidant induced oxidative stress in rats' brain-in vitro. Neurochem Res. 2013;38(2):413-9.

44. Shah $\mathrm{M}$, et al. Chlorogenic acid alleviates neurobehavioral disorders and brain damage in focal ischemia animal models. Neurosci Lett. 2021;760:136085.

45. Middeldorp J, Hol E. GFAP in health and disease. Progress in neurobiology. 2011;93(3):421-43. 
46. Cuesta S, Proietto R, García G. Astrogliosis and HSP 70 activation in neonate rats' brain exposed to sodium metavanadate through lactation. Neurotoxicol Teratol. 2013;37:57-62.

\section{Figures}

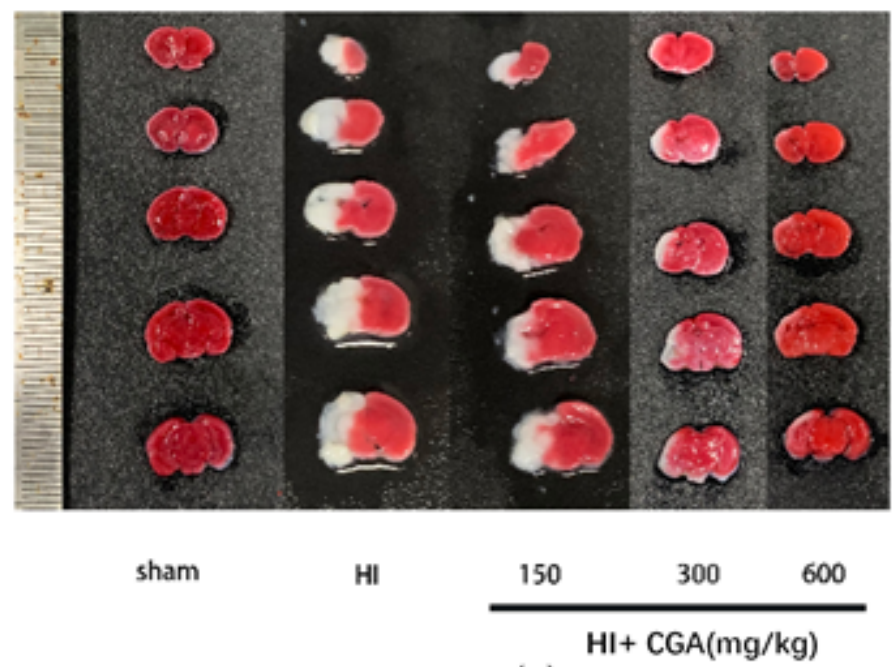

(a)

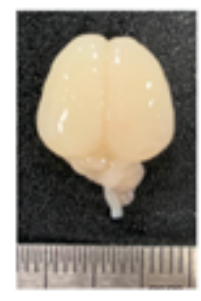

sham

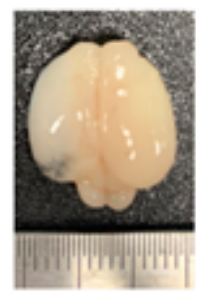

HI

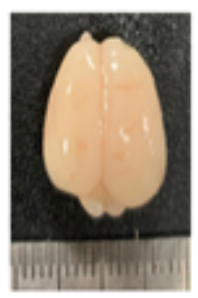

$\mathrm{HI}+\mathrm{CGA}(600 \mathrm{mg} / \mathrm{kg})$

(c)

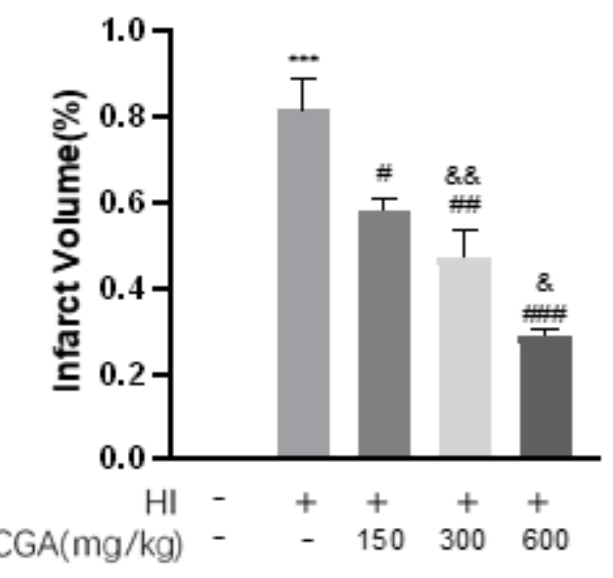

(b)

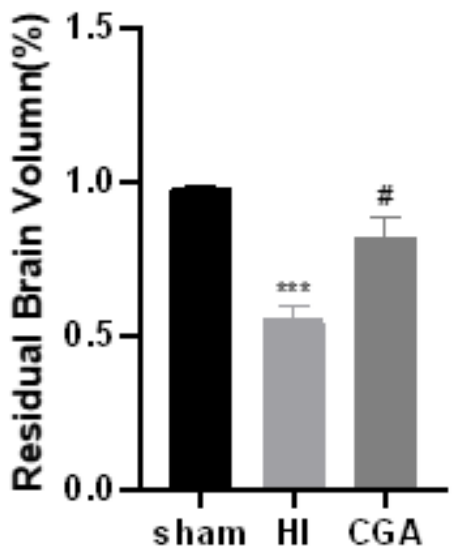

(d)

\section{Figure 1}

Chlorogenic acid-induced attenuation of hypoxic-ischemic brain injury in neonatal rats. (a) Representative images of TTC-stained coronal brain sections $24 \mathrm{~h}$ after $\mathrm{HI}$ brain injury with/without treatments. (b) Calculation of the infarct area depicted by TTC staining. $* * * \mathrm{P}<0.001$ vs. the sham group. \#P $<0.05$, $\# \# P<0.01$ and \#\#\#P< 0.001 vs. the Hl group. \&P $<0.05$, \&\&P $<0.01$ vs. the CGA $(150 \mathrm{mg} / \mathrm{kg})$ group. $\mathrm{n}=$ 4. (c) The brains isolated from each group 7days after $\mathrm{HI}$ brain injury. (d) The ratio of residual brain volume calculated in each group. $* * * P<0.001$ vs. the sham group. $\# \mathrm{P}<0.05$ vs. the HI group. $\mathrm{n}=5$. 


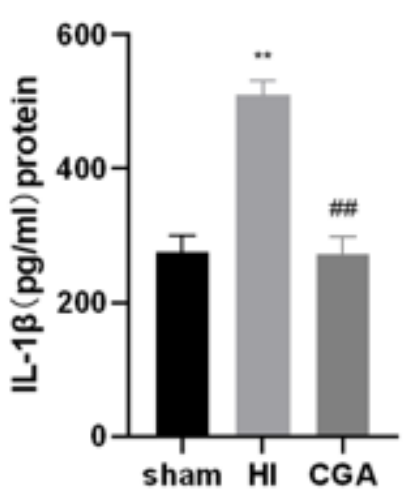

(a)

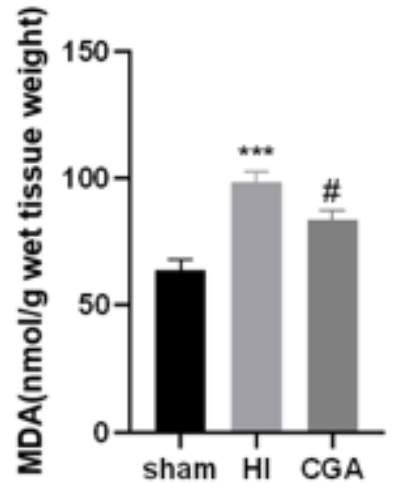

(b)

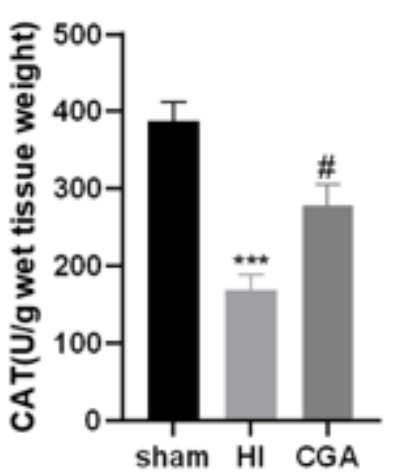

(c)

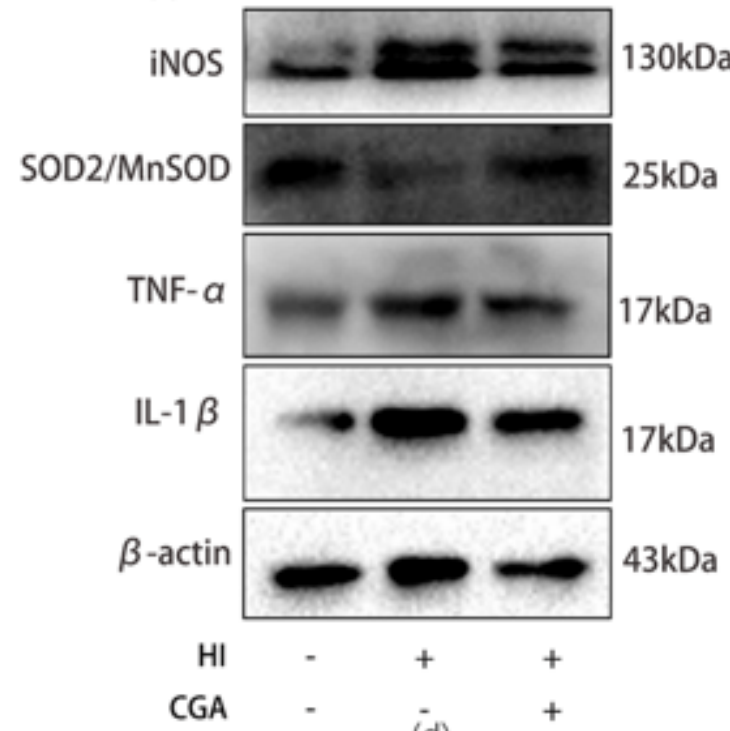

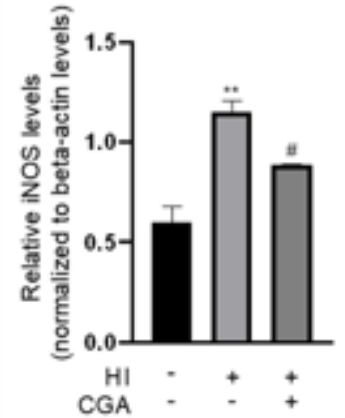

(e)

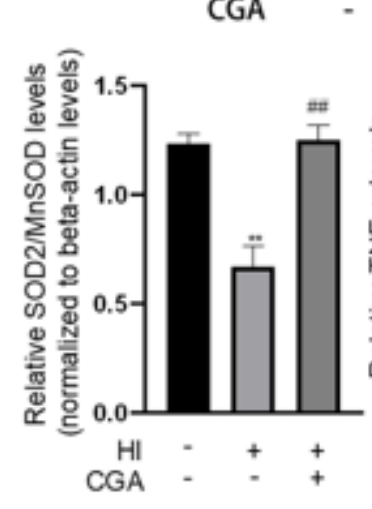

(f)

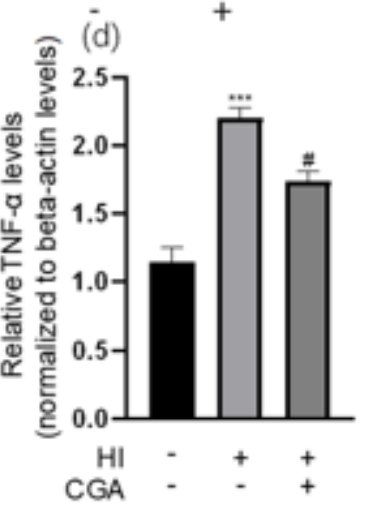

(g)

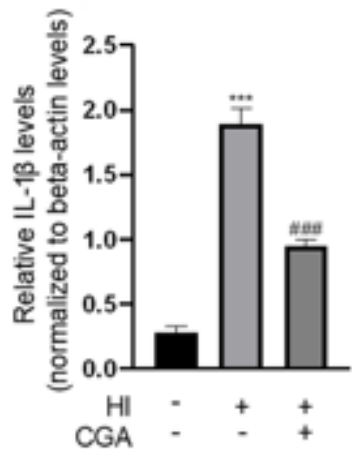

(h)

Figure 2

Chlorogenic acid-induced protection of brain post-hypoxic-ischemic brain injury via the down-regulation of expression of inflammatory and oxidative stress levels. (a) The IL-1 $\beta$ level in brain tissue $24 \mathrm{~h}$ after HI brain injury measured by Elisa kit. $* \mathrm{P}<0.05$ vs. the sham group. \#\#P $<0.01$ vs. the HI group. $\mathrm{n}=3$. (b) The MDA level in brain tissue $24 \mathrm{~h}$ after $\mathrm{HI}$ brain injury by MDA kit. $* * * \mathrm{P}<0.001 \mathrm{vs}$. the sham group. $\# \mathrm{P}<$ 0.05 vs. the HI group. $\mathrm{n}=3$. (c) The CAT level in brain tissue $24 \mathrm{~h}$ after $\mathrm{HI}$ brain injury by CAT kit. $* * * \mathrm{P}<$ 0.001 vs. the sham group. \#P $<0.05$ vs. the HI group. $n=3$. (d) Western blot detection of the protein levels of iNOS, SOD2/MnSOD, TNF-a, and IL-1 $\beta$ 24h after HI injury. (e-h) Quantification of western blot data of 
iNOS, SOD2/MnSOD, TNF-a, and IL-1 $\beta . * * P<0.01$ and $* * * P<0.001$ vs. the sham group. \#P $<0.05$, \#\#P $<0.01$ and \#\#\# $<0.001$ vs. the HI group. $\mathrm{n}=3$.
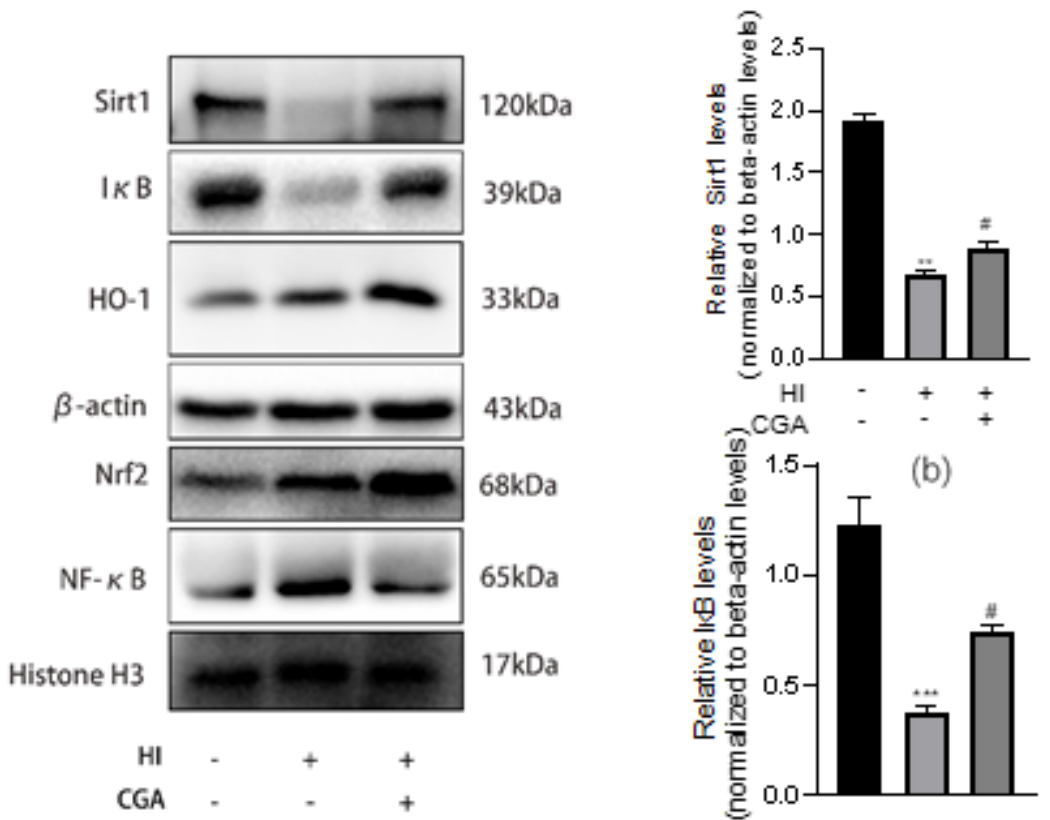

(a)

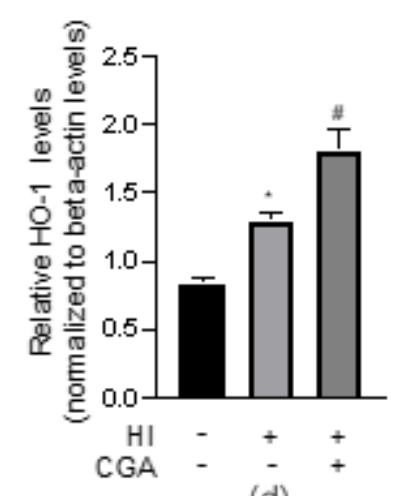

(d)

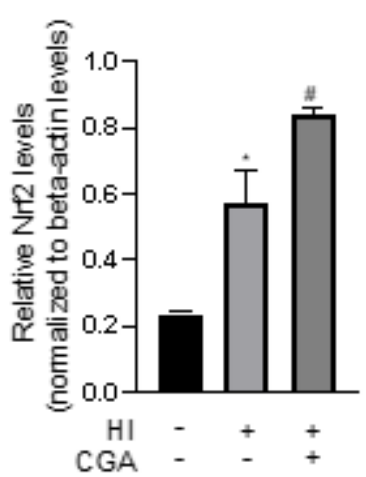

(e)

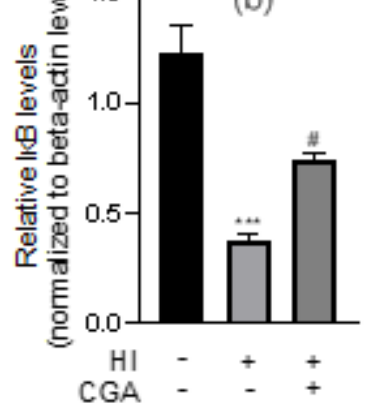

(c)

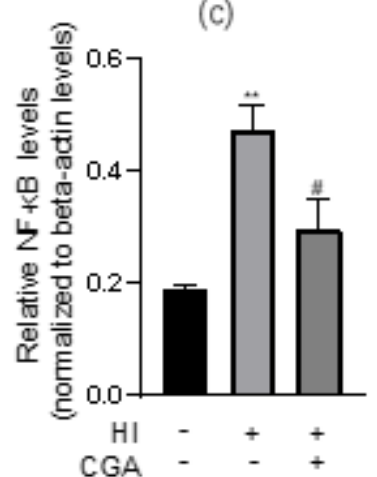

(f)

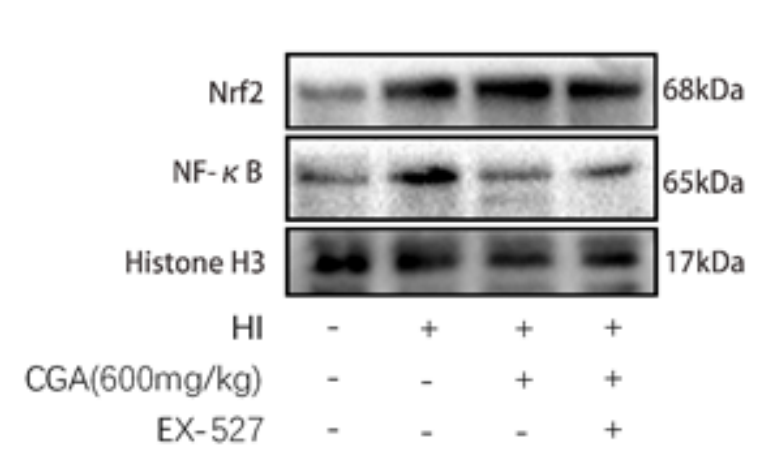

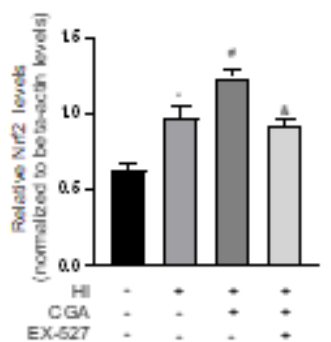

(h)

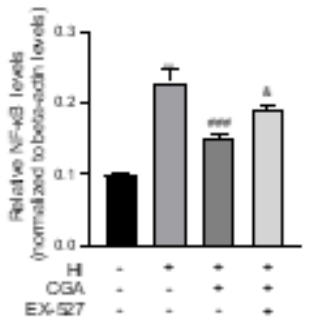

(i)

(g)

Figure 3

The neuroprotective role of CGA in hypoxic-sschemic brain injury through activation of Sirt1 to regulate the Nrf2-NF-KB signaling pathway. (a) Representative results from Western blot showing the protein levels of Sirt1, IkB, and HO-1 in cytoplasm, Nrf2 and NF-kB in nucleus 24h after HI injury. (b-f) Quantification of 
western blot data of Sirt1, IKB, HO-1, Nrf2, NF-KB. $* \mathrm{P}<0.05, * * \mathrm{P}<0.01$ and $* * * \mathrm{P}<0.001$ vs. the sham group. \#P<0.05, \#\#P $<0.01$ and \#\#\#P $<0.001$ vs. the HI group. $\mathrm{n}=3$. (g) Representative results from Western blot showing the protein levels of Nrf2 and NF-KB in nucleus 24h after HI injury. (h-i) Quantification of western blot data of Nrf2, NF-KB. $* \mathrm{P}<0.05, * * \mathrm{P}<0.01$ vs. the sham group. \#P $<0.05$, $\#$ \# $<0.01$ vs. the HI group. \&P $<0.05$, vs. the CGA pretreatment group. $n=3$.

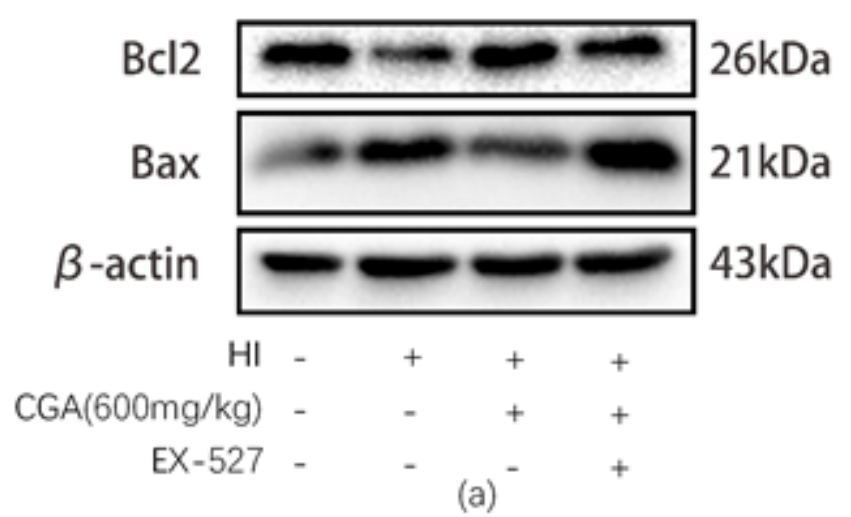

(a)

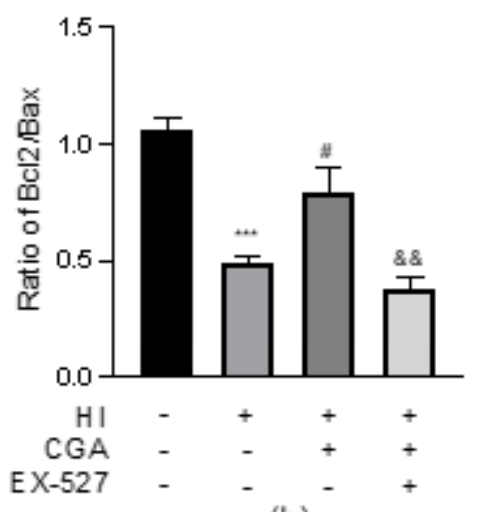

(b)

\section{Figure 4}

Chlorogenic acid (CA) treatment reduces $\mathrm{HI}$-induced neuronal apoptosis. (a) Western blot evaluation of the protein levels of $\mathrm{Bcl} 2$ and $\mathrm{Bax} 24 \mathrm{~h}$ after $\mathrm{HI}$ injury. (b) Quantification of western blot data of $\mathrm{Bcl} 2 / \mathrm{Bax}$. $* * * \mathrm{P}<0.001$ vs. the sham group. $\# \mathrm{P}<0.05$ vs. the $\mathrm{HI}$ group. \&\&P $<0.01$ vs. the CA treatment group. $\mathrm{n}=$ 3. 


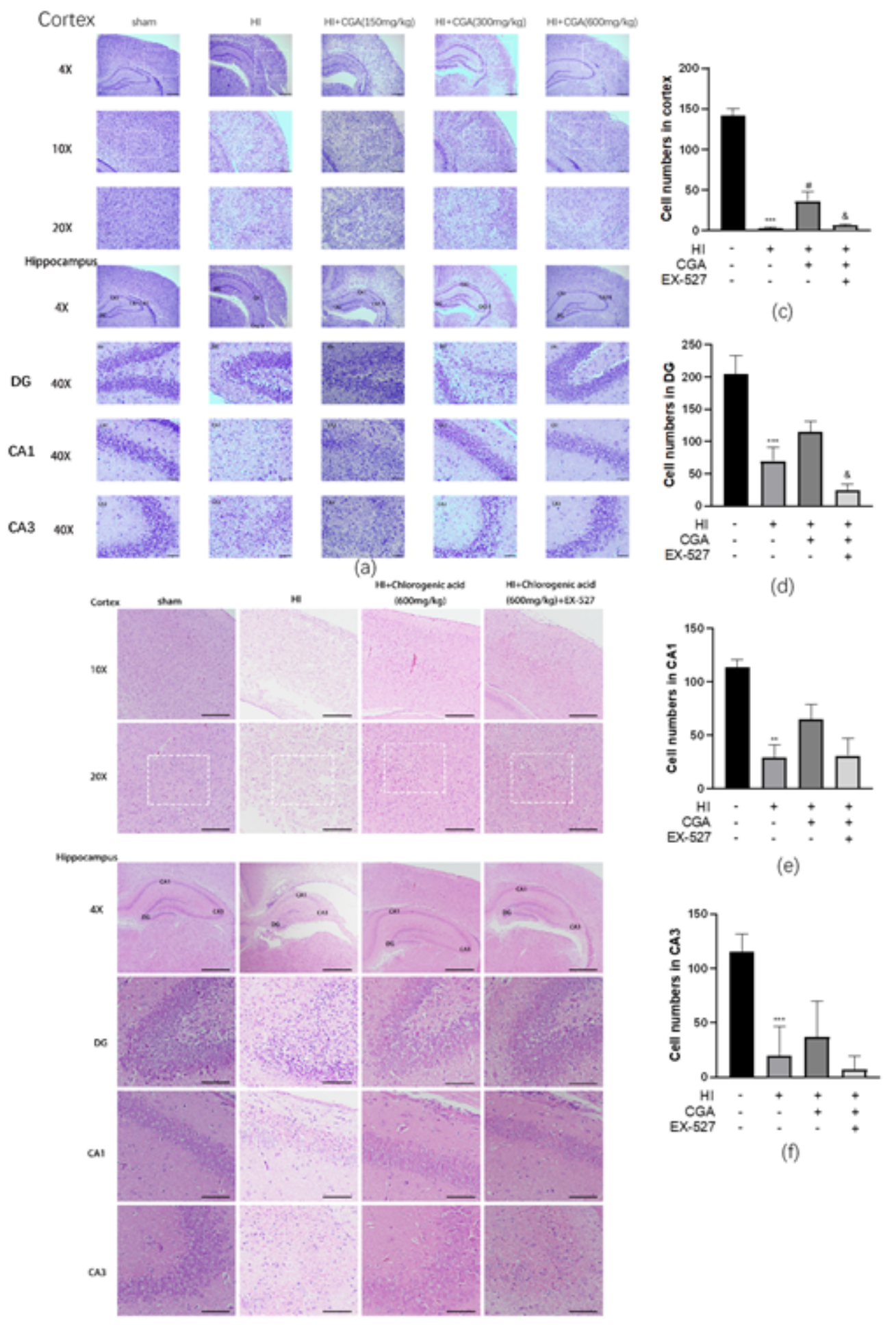

(b)

\section{Figure 5}

Chlorogenic acid improves hypoxic-ischemic brain injury-induced brain tissue structural damage. (a) Representative images of HE staining in the cortex, hippocampus CA1 region, hippocampus CA3 region, and DG region 7 days after $\mathrm{HI}$ injury. Scale bars represent $200 \mu \mathrm{m}$ in the images of $4 X$; Scale bars represent $100 \mu \mathrm{m}$ in the images of 10X; Scale bars represent $50 \mu \mathrm{m}$ in the images of 20X. Scale bars

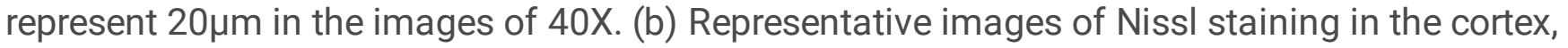


hippocampus $\mathrm{CA} 1$ region, hippocampus $\mathrm{CA} 3$ region, and $\mathrm{DG}$ region 7 days after $\mathrm{HI}$ injury. Scale bars represent $200 \mu \mathrm{m}$ in the images of $4 \mathrm{X}$, Scale bars represent $100 \mu \mathrm{m}$ in the images of $10 \mathrm{X}$, Scale bars

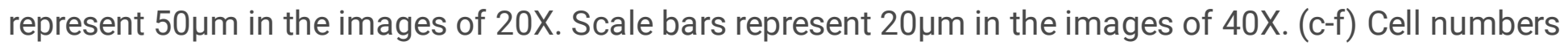
in the cortex of the images of 10X, hippocampus CA1 region, hippocampus CA3 region, and DG region in each group 『results of treatments are based on the $600 \mathrm{mg} / \mathrm{kg}$ of CGA. $* * * P<0.001 \mathrm{vs}$. the sham group. $\# P<0.05$, \#\#P $<0.01$ and \#\#\#P $<0.001$ vs. the HI group. \&P $<0.05, \& \& P<0.01, \& \& \& P<0.001$ vs. the CA treatment group. $\mathrm{n}=3$

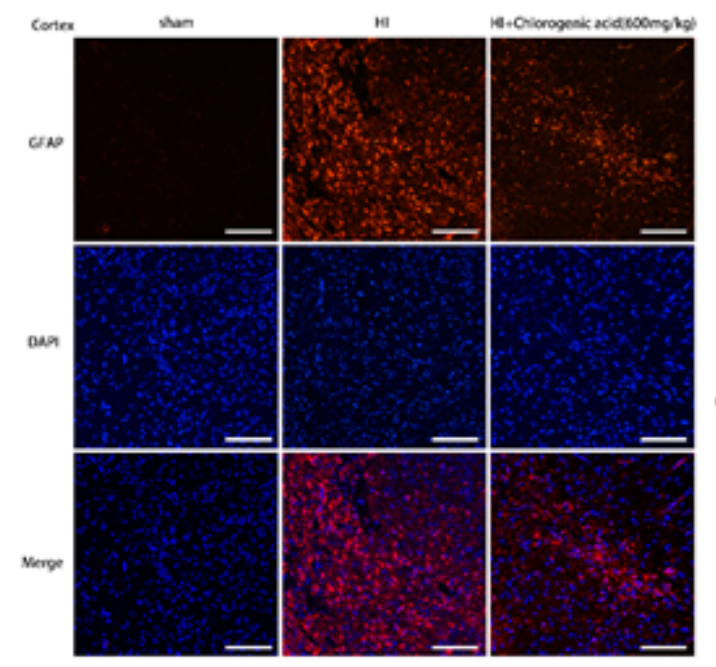

(a)

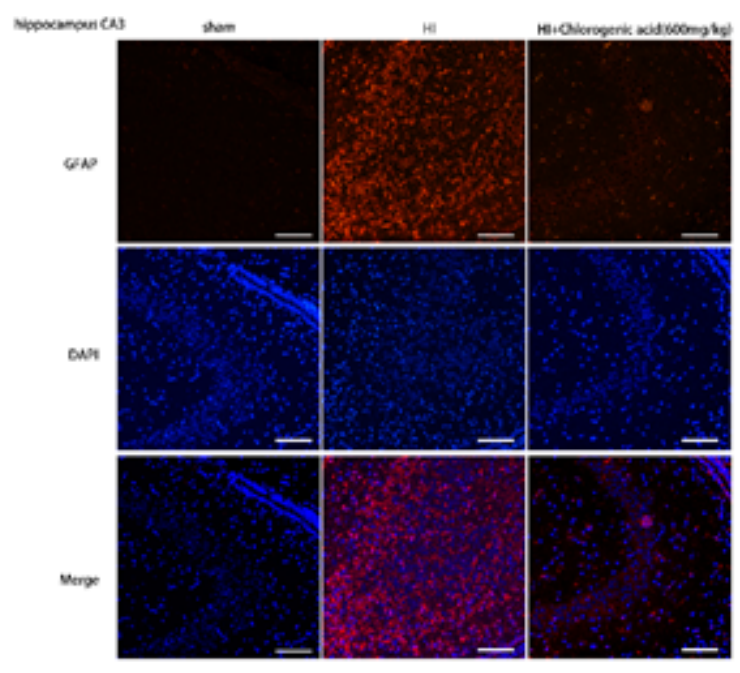

(b)

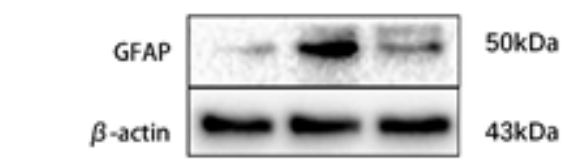

CGA(600mg/kg)

(c)

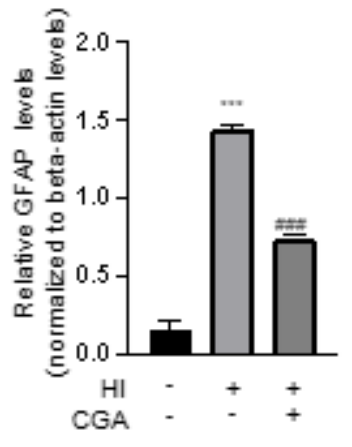

(d)

\section{Figure 6}

Chlorogenic acid treatment suppressed Hl-induced activation of astrocyte in the neonate brain. (a-b) Representative immunofluorescent staining for GFAP expression in the cortex (a) and hippocampus CA3 region (b) from each group at 7days post-HI. Scale bar $=50 \mu \mathrm{m} . \mathrm{n}=3$ (c) Western blot evaluation of the protein levels of GFAP 1 day after HI. (d) Quantification of western blot data of GFAP. $* * * P<0.001$ vs. the control group. \#P< 0.05 vs. the OGD group. $n=3$. 


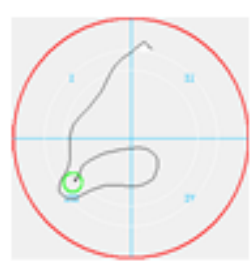

sham

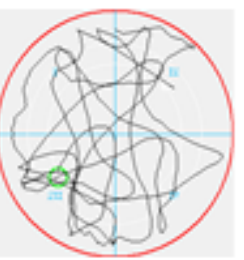

sham

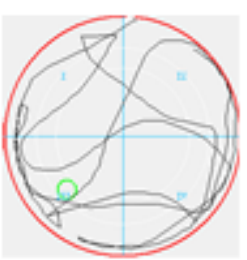

HI

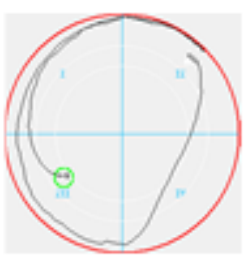

$\mathrm{HI}+\mathrm{CGA}$

(a)

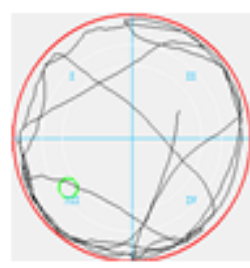

HI

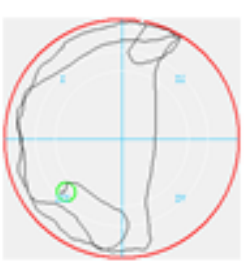

$\mathrm{HI}+\mathrm{CGA}+\mathrm{EX}-527$

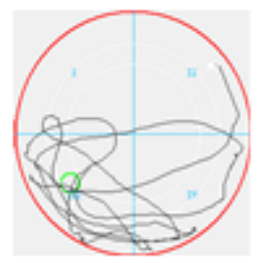

(c)

$\mathrm{HI}+\mathrm{CGA}+\mathrm{EX}-527$

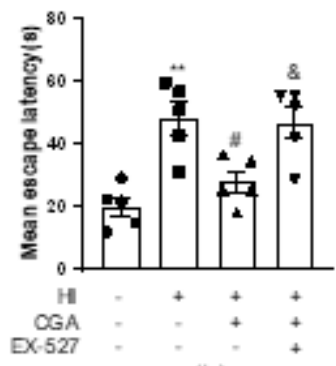

(b)

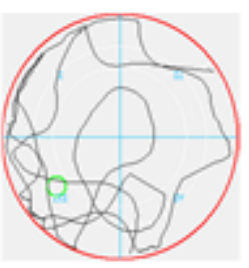

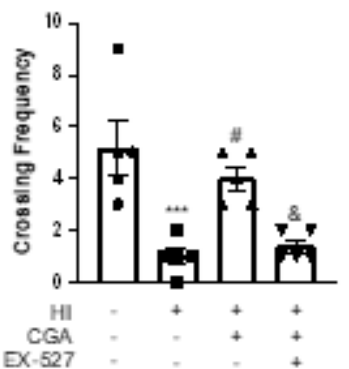

(d)

\section{Figure 7}

Chlorogenic acid ameliorates the long-term learning and cognitive function of rats with hypoxic-ischemic brain injury (a) Representative of swim route traces of rats from different groups. (b) Quantitative analysis of mean escape latency of Morris Water Maze tests in different groups of rats. ${ }^{\star \star} \mathrm{P}<0.01 \mathrm{vs}$. the sham group; \#P< 0.05 vs. the HI group; \&P< 0.05 vs. the $H I+C A$ group. $n=5$. (c) Representative images of swim route traces of rats from different groups after removal of the platform. (d) Quantitative analysis of the frequency of crossing the original platform location in the $60 \mathrm{~s}$. ${ }^{* *} \mathrm{P}<0.001 \mathrm{vs}$. the sham group; $\# \mathrm{P}<0.05$ vs. the HI group; \&P $<0.05$ vs. the $\mathrm{HI}+\mathrm{CA}$ group. $\mathrm{n}=5$. 


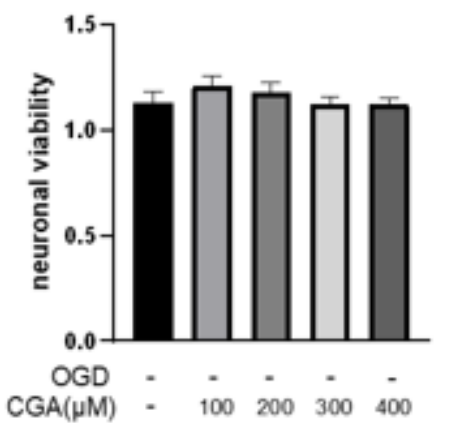

(a)

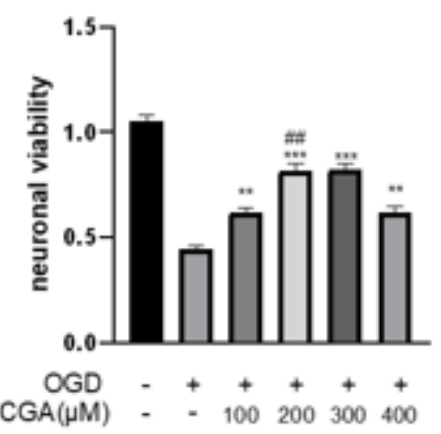

(b)

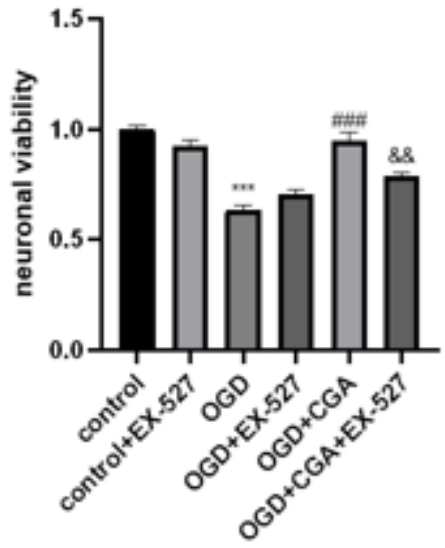

(c)

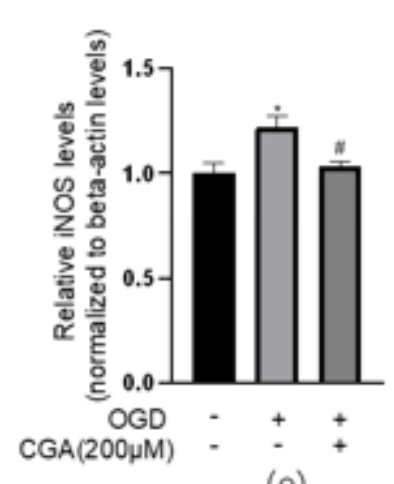

(e)

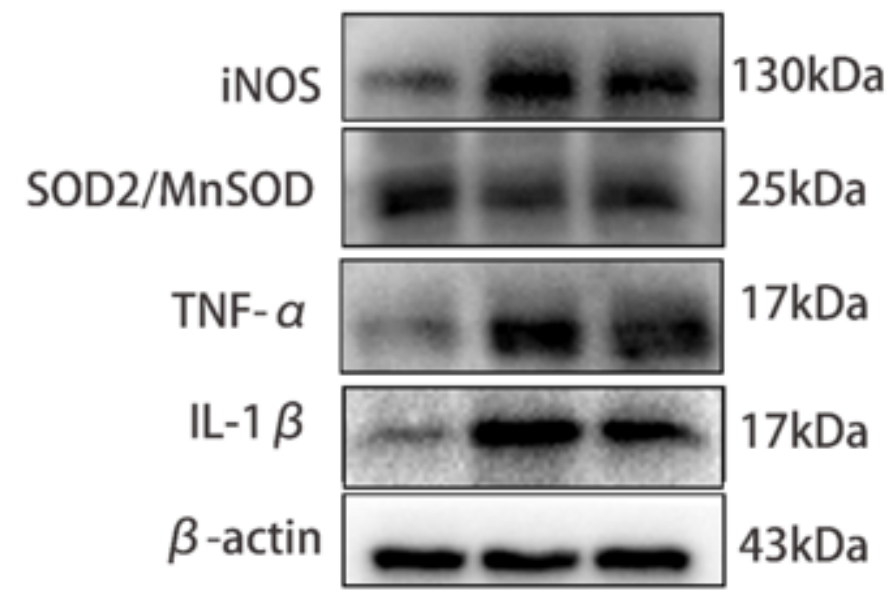

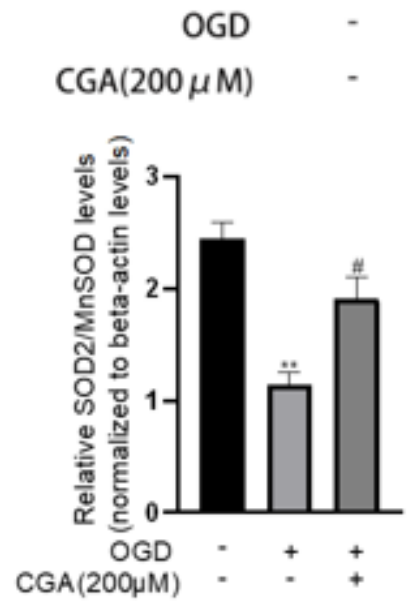

(f)

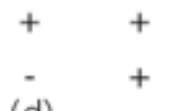

(d)

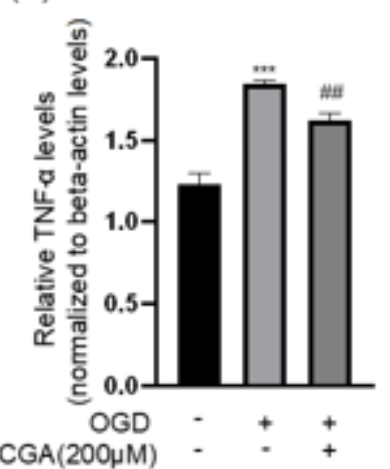

(g)

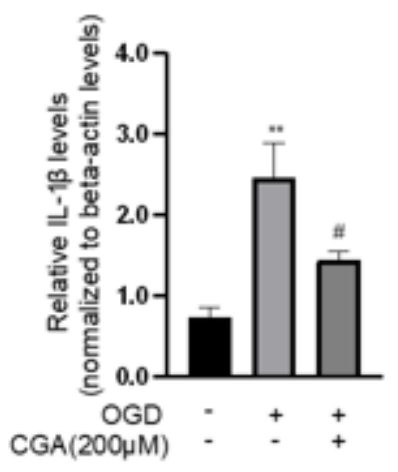

(h)

\section{Figure 8}

Chlorogenic acid decreases inflammation, oxidative stress of primary cortical neurons induced by oxygen and glucose deprivation. (a) Primary cortical neurons, treated with different concentrations of CGA without OGD; cell viability was assessed by CCK8. $n=3$. (b) Primary cortical neurons pretreated with different concentrations of CGA before OGD; cell viability was assessed by CCK8. $n=3$. (c) The effect of Sirt1 inhibitor, EX-527 on neuronal viability $24 \mathrm{~h}$ after OGD for $2 \mathrm{~h} . \mathrm{n}=3$. (d) Western blot evaluation of the protein levels of iNOS, SOD2/MnSOD, TNF-a, and IL-1 $\beta$ 24h after OGD. (e-h) Quantification of western 
blot data of iNOS, SOD2/MnSOD, TNF-a, and IL-1 $\beta . * * \mathrm{P}<0.01$ and $* * * \mathrm{P}<0.001$ vs. the control group. $\# \mathrm{P}<0.05$ and \#\#\#P<0.001 vs. the OGD group. $\mathrm{n}=3$.

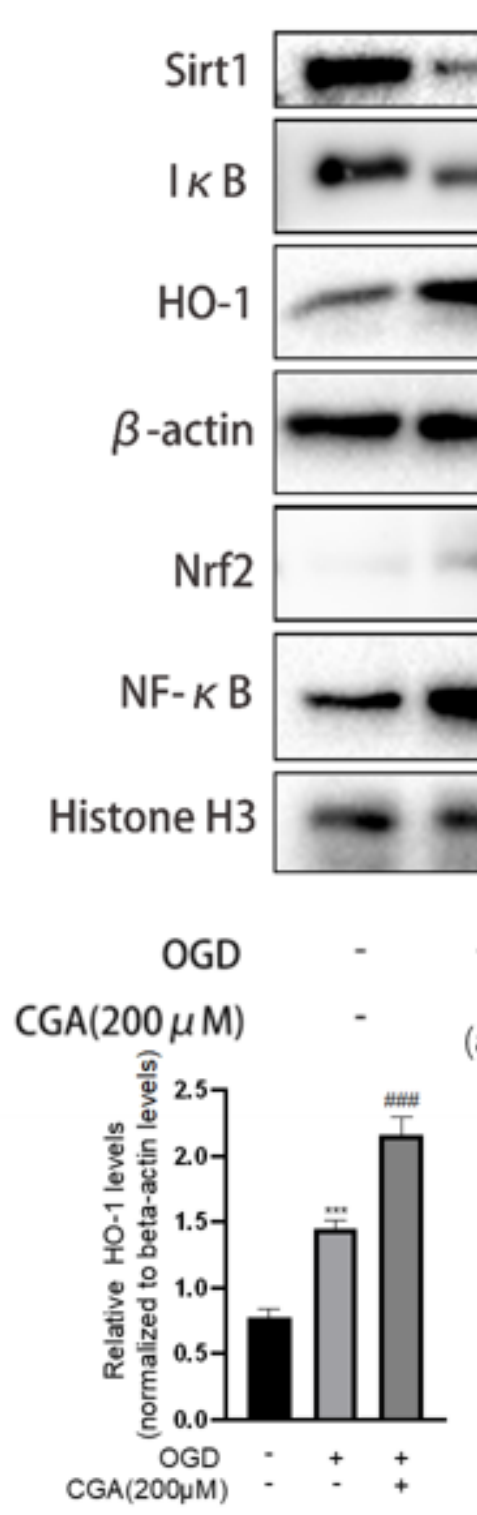

(d)

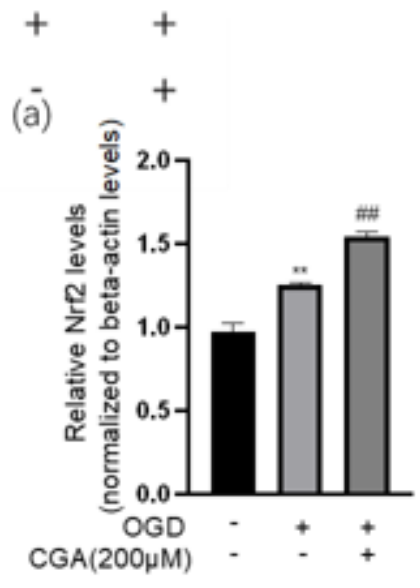

(e)

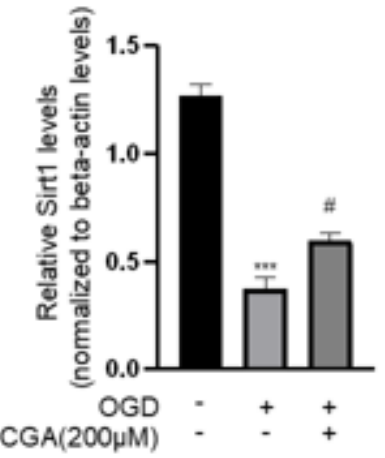

(b)

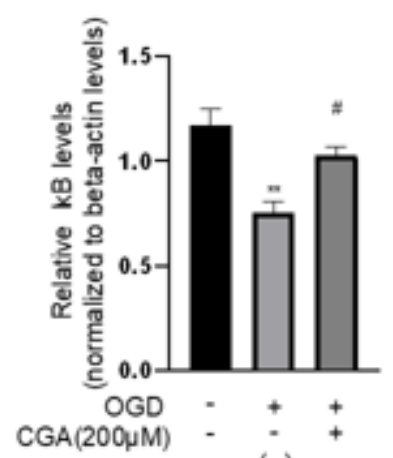

(c)

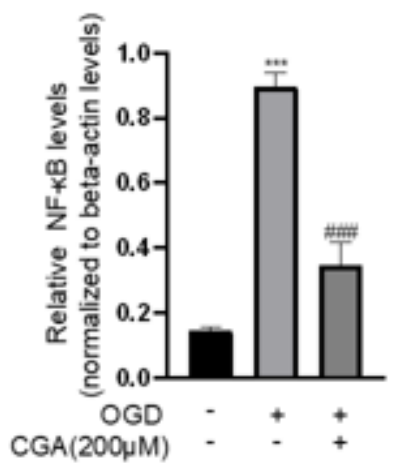

(f)

\section{Figure 9}

Chlorogenic acid regulates the Nrf2-NF-KB signaling pathway by activating Sirt1 in primary cortical neurons. (a) Western blot evaluation of the protein levels of Sirt1, IKB, HO-1, Nrf2, NF-KB 24h after OGD. (b-f) Quantification of western blot data of Sirt1, IKB, HO-1, Nrf2, NF-KB. $* * \mathrm{P}<0.01$ and $* * * \mathrm{P}<0.001$ vs. the control group. $\# \mathrm{P}<0.05, \# \# \mathrm{P}<0.01$ and $\# \# \# \mathrm{P}<0.001$ vs. the OGD group. $\mathrm{n}=3$. 